\title{
EDITORIAL
}

\section{JORNADA «LA ATENCIÓN A LA SALUD MENTAL EN ESPAÑA Y EL OBSERVATORIO DE LA ASOCIACIÓN ESPAÑOLA DE NEUROPSIQUIATRÍA»}

El 18 de octubre de 2005 se celebró en Madrid la jornada de presentación del Observatorio de Salud Mental de la Asociación Española de Neuropsiquiatría, con la colaboración del Ministerio de Sanidad y Consumo y la participación de la práctica totalidad de responsables de salud mental autonómicos, a quienes agradecemos desde estas páginas su excelente disposición. El acto inaugural estuvo a cargo de D. Enrique Terol (en representación del Secretario General del Ministerio) y del presidente de la AEN. La elaboración del Observatorio de Salud Mental es probablemente una de las acciones más reseñables de la AEN en los últimos años, y en este sentido es de justicia felicitar a la anterior junta directiva como promotora del proyecto, así como a Fermín Pérez - primer coordinador-, Isabel Salvador -actual responsable-, y a cuantos han colaborado desde las diferentes asociaciones autonómicas. En el apartado «Páginas de la Asociación» de este número de la revista se incluye información detallada sobre el desarrollo de la jornada, participantes, y conclusiones, pero hay algunos aspectos generales que quizás valga la pena reseñar:

Aún reconociendo los avances innegables que se han producido en nuestro país en los últimos veinte años en la atención a la salud mental -tanto en la dotación de recursos como en la implantación de programas específicos-, la situación, tanto a nivel estatal como autonómico ${ }^{1}$, dista mucho de ser la que correspondería por nivel socioeconómico, y está muy alejada de lo planteado cuando se iniciaron los movimientos de reforma psiquiátrica. Es curioso -y en este aspecto la colección de Revistas de la $\mathrm{AEN}^{2}$ es una fuente inagotable de conocimiento sobre la evolución de la asistencia psiquiátrica en España- que ya en 1950 (Congreso de Valencia) la AEN señalara «la desigualdad que se observa en las provincias españolas en la asistencia psiquiátrica...». Ha pasado el tiempo y la Ley de Cohesión y Calidad del Sistema Nacional de Salud propone «el análisis permanente mediante estudios comparados de los servicios de salud de las comunidades autónomas». Distintas palabras, pero idénticos objetivos... incumplidos al día de hoy.

La bondad del modelo de atención preconizado por todas las Comunidades Autónomas (psiquiatría comunitaria) ya no es discutida, pero la realidad es que en muy pocas se ha llevado su desarrollo hasta el final, habiéndose limitado a experiencias parciales (Andalucía, Madrid, Asturias...). La valoración global de los nuevos servicios 
de salud mental permite apreciar su dimensión comunitaria en la medida en que cumplen los criterios de proximidad (centros accesibles), normalización (hospitalización en hospitales generales), y una cierta diversificación (dispositivos y programas según edad, tipo de problemas, gravedad, momento evolutivo, contexto). Sin embargo, se cumplen en mucha menor medida otros criterios, y son prácticamente inexistentes los programas de intervención comunitaria, y de intervención a domicilio.

Aunque la Ley General de Sanidad indicaba que la hospitalización de casos agudos debe hacerse en los hospitales generales, al día de hoy siguen existiendo unidades de agudos en los hospitales psiquiátricos... que fueron cerrados en algunas comunidades (Andalucía), pero siguen siendo un recurso principal en otras (Cataluña, País Vasco, Madrid).

La evolución de la demanda (cuantitativa y cualitativa) no ha ido acompañada de un aumento de recursos proporcional, por lo que sería imprescindible acometer una adecuación de las plantillas, tanto en número como en cualificación. En este sentido es especialmente insuficiente el número de psicólogos clínicos que trabajan en al sistema público de salud ${ }^{3}$. Al mismo tiempo es preciso establecer carteras de servicios que permitan conocer, tanto a los usuarios como a los profesionales, las prestaciones disponibles e incluso la priorización de las mismas.

Casi todos los Servicios de Salud de las Comunidades Autónomas han redactado Planes de Salud Mental que en la mayoría de los casos se han quedado aparcados. Es imprescindible que estos planes vayan acompañados de la correspondiente financiación y cronograma si no se quiere que queden en una mera declaración de intenciones, frustrándose así una y otra vez las expectativas de profesionales y usuarios.

Hay colectivos especialmente desatendidos: Mientras que en los últimos años se ha progresado en la atención al trastorno mental severo (programas de rehabilitación psicosocial, equipos de seguimiento, etc.) en la atención infantojuvenil y en psicogeriatría sigue siendo notable -en algunos casos, escandalosa- la carencia de recursos. Por ejemplo, a pesar de que por ley los niños y adolescentes que necesiten ingreso psiquiátrico deben hacerlo en unidades específicas, en varias CC.AA. siguen haciéndolo en unidades de adultos. En fin, que es mucho lo avanzado pero mucho también lo que queda por hacer.

Y para terminar, un mensaje de tono nostálgico-optimista: Es cierto que no se han cumplido en su totalidad las expectativas creadas por una generación de profesionales que se empeñaron en dignificar la atención a los enfermos mentales, pero no cabe duda de que de la situación de partida a la actual media un abismo. Después de veinte años del inicio de la reforma psiquiátrica se abren nuevas oportunidades: La «Conferencia de Helsinki» y su Plan de Salud Mental para Europa, el grupo de trabajo «Estrategias en Salud Mental», propiciado por el Ministerio de Sanidad y en el que participan varios compañeros de la AEN, el desbloqueo del Consejo Interterritorial con la posibilidad de llegar a acuerdos de conjunción entre las Consejerías de Salud 
de las CC.AA, etc., son muestras de que las autoridades sanitarias han retomado la sensibilidad por los problemas de salud mental de la población. Que esa sensibilidad se traduzca en acciones es lo que está por ver. Sin falsos optimismos, pero sin derrotismos a priori, la AEN continuará colaborando en el mantenimiento de un sistema público de salud, de calidad, comunitario y equitativo, que ha sido desde el inicio de su andadura su principal seña de identidad.

Francisco Chicharro (Presidente de la AEN)

1. El desarrollo de la reforma psiquiátrica (avalada por la Ley General de Sanidad, art. 20- publicada en 1986) ha sido desigual, tanto en las diferentes comunidades autónomas como dentro de cada una de las mismas.

2. Revista de la $A E N n^{0}$ 75; 2002 (número monográfico): «Historia de la Asociación Española de Neuropsiquiatría».

3. Mientras que en la UE, el número de psiquiatras/100.000 habitantes es de 10, en España no llega a 6 . En cuanto a los psicólogos, en la UE es de 19,6 y en España 4,3. 